\title{
Isotropic and Nonisotropic Components of Earthquakes and Nuclear Explosions on the Lop Nor Test Site, China
}

\author{
B. G. Bukchin, ${ }^{1}$ A. Z. Mostinsky, ${ }^{1}$ A. A. Egorkin, ${ }^{1}$ A. L. Levshin, ${ }^{2}$ \\ and M. H. RITZWOLLER ${ }^{2}$
}

\begin{abstract}
We test the hypothesis that the existence of an observable non-zero isotropic component of seismic moment can be used as a discriminant to distinguish nuclear explosions from shallow earthquakes. We do this by applying the method described herein to a small set of data recorded between 1990 and 1996 following events (seven nuclear explosions, three earthquakes) that occurred on the Lop Nor test site in Western China. We represent each source as a sum of an isotropic component at the surface and a nonisotropic, double-couple component at an estimated depth. The explosions all possess a significant nonzero isotropic component and the estimated depth of the double-couple component of the moment tensor, presumably the result of tectonic release, lies between about 0 and $3 \mathrm{~km}$. For the earthquakes studied, the isotropic component is indistinguishable from zero and the depths of the sources are estimated at 3,17 and $31 \mathrm{~km}$. The data set we have studied, although still very small, suggests that certain source characteristics (namely, double-couple depth and the ratio of the isotropic to nonisotropic components of seismic moment) may prove useful in discriminating explosions from shallow earthquakes. Further work is needed to determine whether these observations hold for explosions at other test sites, to investigate a much larger set of shallow earthquakes located in regions of interest, and to study the robustness of the estimated source parameters as source magnitude and the number of observing stations decrease.
\end{abstract}

Key words: Tectonic release, seismic moment tensor, nuclear explosions, earthquakes, surface waves.

\section{Introduction}

It has long been known that nearly all underground nuclear explosions have a significant component of nonisotropic seismic radiation (e.g., Press and ARCHAMBeAu, 1962; Brune and Pomeroy, 1963; AKi and Tsai, 1972; Toksöz and Kehrer, 1972; Helle and RygG, 1984; Wallace et al., 1985; Walter and Patton, 1990). There appears to be general agreement that the long-period part of the nonisotropic radiation is caused by tectonic release due to existing tectonic stresses in the source region (Mueller and Murphy, 1971; Archambeau, 1972; Masse, 1981; Wallace

\footnotetext{
${ }^{1}$ International Institute of Earthquake Prediction Theory \& Mathematical Geophysics, Moscow, Russia. E-mail: bukchin@mitp.ru

${ }^{2}$ Department of Physics, University of Colorado, Boulder, Boulder, CO 80309-0390, USA.

E-mail: levshin@lemond.colorado.edu, web: ciei.colorado.edu
} 
et al., 1985; Day and Stevens, 1986; Stevens, 1986; Day et al., 1987; PAtTon, 1991; STEVEns et al., 1991; HARKRIDER et al., 1994; EKSTRöM and RichARDS, 1994; Li et al., 1995).

Most of the experimental studies of tectonic release have concentrated on explosions at the Nevada test site and the test sites in Kazakhstan. Burger et al. (1986) described the long-period $S$-wave radiation by tectonic release of explosions at Novaya Zemlya. Zhang (1994), Levshin and Ritzwoller (1995), and Wallace and Tinker (1996) studied Chinese nuclear explosions and found that most are accompanied by tectonic release. However, we are unaware of any detailed analysis of source mechanisms of these events.

Several authors have estimated the relative contributions of the nonisotropic (tectonic release) and isotropic (explosion) components to the resulting seismic moment tensor of explosions (see, for example, EKSTRöM and RichARDS, 1994). As a rule these estimates have been performed using the assumption that both phenomena occur in the same small volume inside the earth.

Although the determination of the source mechanism of earthquakes is a routine operation, only a few studies have evaluated the isotropic component of earthquake radiation. This is especially true for weak events that are of the primary interest for discrimination purposes. As is well known, a pure shear dislocation does not radiate an isotropic component. Subsequently, the natural tendency is to constrain the moment tensor solution with the assumption of a zero trace. However, from the point of view of discrimination it is important to determine if a significant isotropic component could appear in unconstrained moment tensor solutions due to physical phenomena in the source region or due to inaccuracy of measurements and underlying assumptions.

In this paper we will describe an approach to estimate the isotropic and nonisotropic components of the source mechanism and will apply this method to a small set of data for several earthquakes and explosions on the Lop Nor test site. The purpose is to determine whether the existence of a strong isotropic moment is consistent with the hypothesis that the event is an explosion. For each of the ten events we consider (7 explosions and 3 earthquakes, Fig. 1), we obtain estimates of the isotropic and nonisotropic moments, characterize the tectonic release by estimating the moment tensor of the nonisotropic source, and estimate the depth of the equivalent double-couple of the nonisotropic source. Our approach differs from those of previous studies in three main ways which are designed to focus the method on the discrimination problem: (1) we allow the depth of tectonic release for all events (earthquakes and explosions) to be variable and fix only the depth of the isotropic source near the surface, (2) we fit only the amplitude of the surface wave spectrum so the method, in principal, can be applied to considerably smaller events for which the interpretation of phase may be ambiguous, and (3) we minimize the misfit between synthetic and observed surface wave amplitude spectra jointly with fitting polarities of $P$-wave first arrivals. 


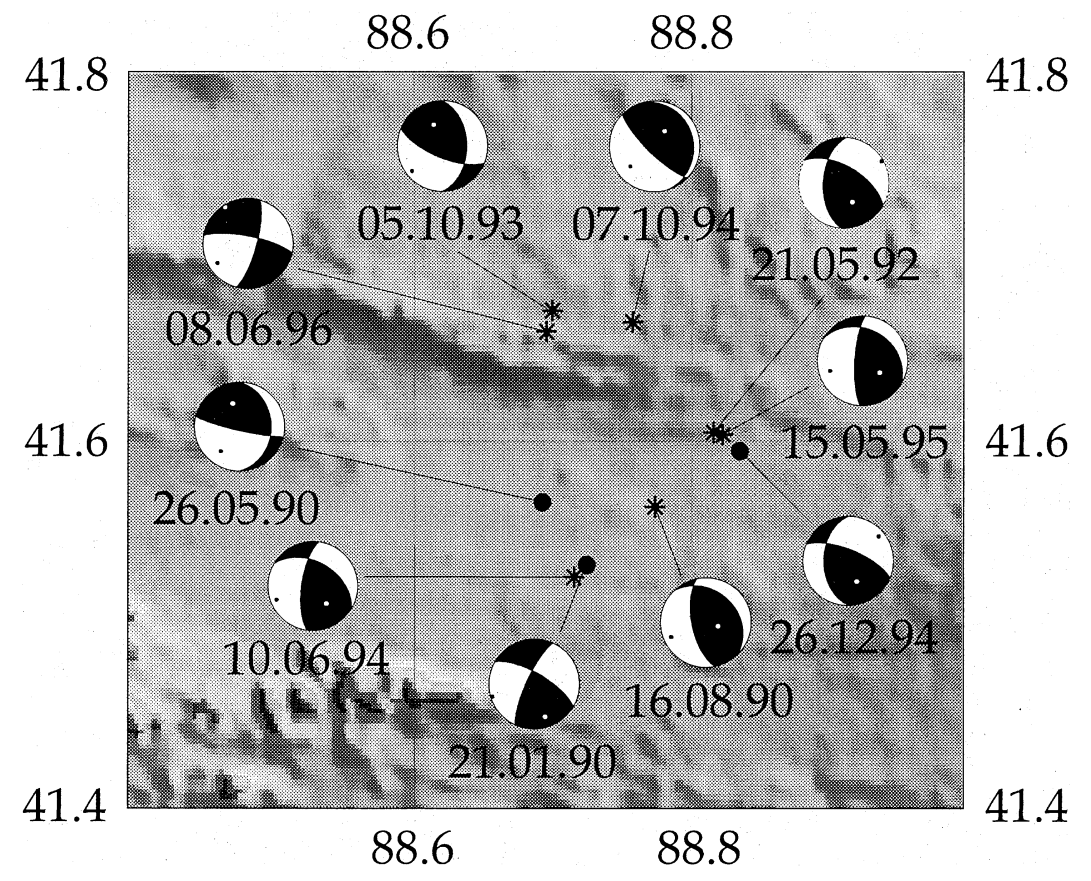

Figure 1

Epicenters and focal mechanisms of earthquakes (circles) and tectonic releases accompanying explosions (stars).

Our approach is, therefore, based on the combination of an isotropic moment tensor at the surface, which models an explosive source, and a pure double-couple at an estimated depth, which models tectonic release. We find it useful to express the seismic moment of the double-couple component, $M_{0 q u}$, and the seismic moment of the isotropic component, $M_{0 e x}$, as the total seismic moment $M_{0}$ and an angle $\varphi$ which determines the ratio of the seismic moments of the isotropic and double-couple components:

$$
\begin{gathered}
M_{0 q u}=M_{0} \cos \varphi \\
M_{0 e x}=M_{0} \sin \varphi \\
\tan \varphi=\frac{M_{0 e x}}{M_{0 q u}} .
\end{gathered}
$$

We call $\varphi$ the isotropic angle, for want of a better term, because $\varphi=0$ corresponds to a pure earthquake and $\varphi=90^{\circ}$ corresponds to a pure explosion. With this notation and the assumption that the isotropic component of the source occurs at a known depth (usually at or near the surface), the source can then be characterized by six parameters: the seismic moment $M_{0}$, the isotropic angle $\varphi$, three angles (or two 
principal axes, $\mathbf{T}$ (tension) and $\mathbf{P}$ (compression)) determining the double-couple focal mechanism, and the double-couple depth $h$.

We determine these source parameters by fitting the polarities of $P$-wave first arrivals jointly while minimizing the misfit between synthetic and observed Love and Rayleigh surface wave amplitude spectra. We search for the optimal set of parameters by a systematic exploration of parameter space. To characterize the uncertainty of the source parameters, we calculate partial residual functions which describe the minimum misfit as a function of each varying parameter.

\section{The Algorithm}

An instantaneous point source can be described by the moment tensor, a symmetric $3 \times 3$ matrix $\mathbf{M}$. Seismic moment, $M_{0}$, is defined by the equation $M_{0}=\left(1 / 2 \operatorname{tr}\left(\mathbf{M}^{T} \mathbf{M}\right)\right)^{1 / 2}$, where $\mathbf{M}^{T}$ is the transposed moment tensor and $\operatorname{tr}\left(\mathbf{M}^{T} \mathbf{M}\right)=\sum_{i, j=1}^{3} M_{i j}^{2}$. The moment tensor for any event can be presented in the form $\mathbf{M}=M_{0} \mathbf{m}$, where the matrix $\mathbf{m}$ is normalized by the condition $\operatorname{tr}\left(\mathbf{m}^{T} \mathbf{m}\right)=2$. We consider each event as a sum of an earthquake (0-trace moment tensor $\left.\mathbf{M}_{q u}\right)$ and an explosion (moment tensor $\mathbf{M}_{e x}$ ). The moment tensor $\mathbf{M}$ of such an event is given by the sum $\mathbf{M}=\mathbf{M}_{q u}+\mathbf{M}_{e x}$. If $\mathbf{I}$ be the $3 \times 3$ identity matrix, then $\mathbf{M}_{e x}=\sqrt{2 / 3} M_{0 e x} \mathbf{I}$, where $M_{0 e x}$ is the seismic moment of the explosion. For the earthquake, $\mathbf{M}_{q u}=M_{0 q u} \mathbf{m}$, where $M_{0 q u}$ is the seismic moment of the earthquake, and $\mathbf{m}$ is a normalized moment tensor that we will assume has the following characteristics: $\operatorname{tr} \mathbf{m}=0$ and $\operatorname{tr}\left(\mathbf{m}^{T} \mathbf{m}\right)=2$. Thus, $\mathbf{m}$ represents a normalized double-couple mechanism and we assume that $\mathbf{M}_{q u}$ represents a double-couple source.

The tensors $\mathbf{M}_{e x}$ and $\mathbf{M}_{q u}$ are orthogonal in the sense we describe in the next paragraph. Thus, the total moment tensor is the sum of two orthogonal components, and the seismic moments of the explosion and earthquake components of the total moment can be expressed uniquely as total seismic moment $M_{0}$ and the isotropic angle $\varphi$ between $\mathbf{M}$ and $\mathbf{M}_{q u}$, as given by equations (1)-(3) above.

To show that $\mathbf{M}_{e x}$ and $\mathbf{M}_{q u}$ are orthogonal, consider the six dimensional linear Euclidean space of symmetric $3 \times 3$ matrices $\mathbf{M}$, and let the scalar product of two vectors $(\mathbf{M}, \mathbf{N})$ be defined as $(\mathbf{M}, \mathbf{N})=\sum_{i, j} M_{i j} N_{i j}=\operatorname{tr}\left(\mathbf{M}^{T} \mathbf{N}\right)$. The isotropic tensors $\mathbf{M}_{e x}$ form a 1-D subspace which is orthogonal to the 5-D linear subspace of zero trace tensors $\mathbf{M}_{q u}$ :

$$
\left(\mathbf{M}_{q u}, \mathbf{M}_{e x}\right)=\sqrt{2 / 3} M_{0 q u} M_{0 e x} \operatorname{tr}\left(\mathbf{m}^{T} \mathbf{I}\right)=\sqrt{2 / 3} M_{0 q u} M_{0 e x} \operatorname{tr} \mathbf{m}=0 .
$$

Finally, the seismic moment of the combined event can be expressed by the formula: 
Love amplitude spectra as function of period

TLY Az= 40.5
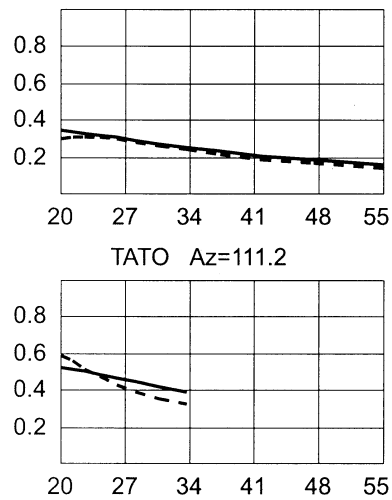

ABKT $\quad A z=271.1$

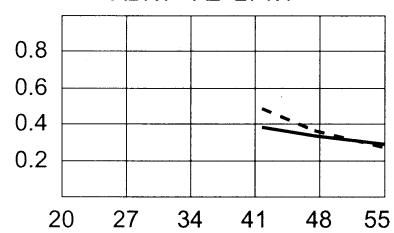

INU $A z=83.3$
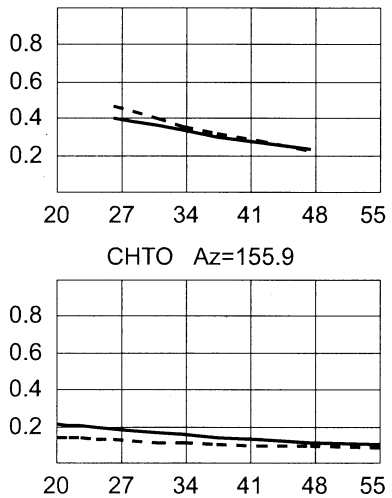

NRIL $\quad A z=359.8$

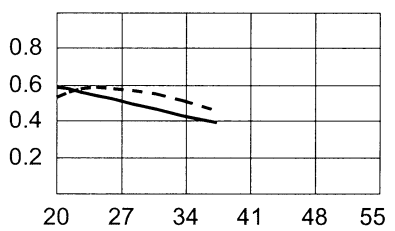

Rayleigh amplitude spectra as function of period

TLY Az= 40.5

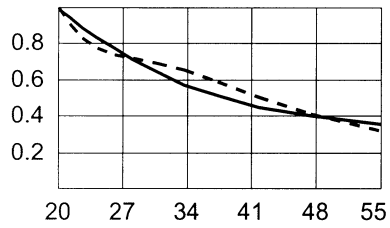

CHTO $\quad A z=155.9$

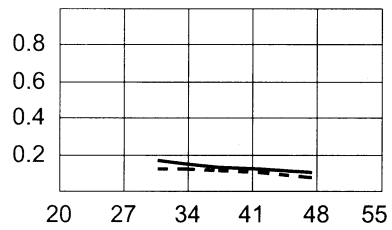

INU $\mathrm{Az}=83.3$

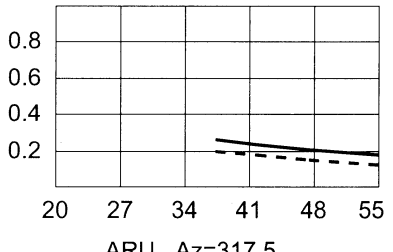

ARU Az=317.5

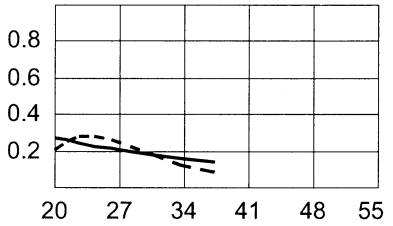

Figure 2

Comparison of observed (dashed lines) and computed (solid lines) Rayleigh and Love wave amplitude spectra for the explosion on 5 October, 1993. Stations' names and azimuths from the epicenter to the stations are provided. All spectra are normalized by the same value.

$$
\begin{aligned}
M_{0} & =\sqrt{1 / 2(\mathbf{M}, \mathbf{M})}=\sqrt{1 / 2\left(\left(\mathbf{M}_{q u}+\mathbf{M}_{e x}\right),\left(\mathbf{M}_{q u}+\mathbf{M}_{e x}\right)\right)} \\
& =\sqrt{1 / 2\left(\mathbf{M}_{q u}, \mathbf{M}_{q u}\right)+1 / 2\left(\mathbf{M}_{e x}, \mathbf{M}_{e x}\right)} \\
& =\sqrt{M_{0 q u}^{2}+M_{0 e x}^{2}} .
\end{aligned}
$$


Our appoach then is to consider a seismic source as the combination of an isotropic tensor, modeling an explosion located at zero depth, and a pure double-couple point source at a depth $h$, modeling the tectonic release. Both explosions and earthquakes are considered as instantaneous sources. Such a source can be described by six parameters: isotropic angle $\varphi$, double-couple depth $h$, three angles (strike, dip, slip) characterizing the focal mechanism or alternately the principal tension and compression axes $(\mathbf{T}, \mathbf{P})$, and the seismic moment $M_{0}$. We determine five of these parameters by a systematic exploration of the five-dimensional parametric space, and the sixth parameter, $M_{0}$, by the least-squares minimization of the misfit between the observed and calculated surface wave amplitude spectra for every current combination of the other parameters.

Under the assumptions mentioned above, the relation between the surface wave displacement spectrum $u_{i}(\mathbf{x}, \omega)$ and the total moment tensor $\mathbf{M}$ can be expressed by the following formula

$$
u_{i}(\mathbf{x}, \omega)=\frac{1}{i \omega}\left[M_{q u_{i l}} \frac{\partial}{\partial y_{q u_{l}}} G_{i j}\left(\mathbf{x}, \mathbf{y}_{q u}, \omega\right)+M_{e x_{j l}} \frac{\partial}{\partial y_{e x_{l}}} G_{i j}\left(\mathbf{x}, \mathbf{y}_{e x}, \omega\right)\right],
$$

where $i, j=1,2,3$ and the summation convention for repeated indices is used. Here $G_{i j}(\mathbf{x}, \mathbf{y}, \omega)$ is the spectrum of the Green's function for the chosen model of the medium and the wave type (see Levshin, 1985; Bukchin, 1990), $\mathbf{y}$ is the source location, and $\mathbf{x}$ is the observation point. We assume that the explosion and earthquake have the same horizontal coordinates, but different depths: $h$ for the earthquake and 0 for the explosion. Another important assumption is that the propagation medium has only weak lateral inhomogeneities so that the surface wave part of the Green's function is determined by structure near the source and receiver, by the average phase velocity along the path, and by geometrical spreading (Woodhouse, 1974; BABICH et al., 1976; Levshin, 1985; LeVshin et al., 1989). The amplitude spectrum $\left|u_{i}(\mathbf{x}, \omega)\right|$ defined by equation (6) does not depend on the average phase velocity of the wave. Moreover, if lateral heterogeneities are sufficiently smooth, errors in source location do not affect the amplitude spectrum (BUKCHIN, 1990). Consequently we use only the amplitude spectra of surface waves to determine the source parameters. An example of fit to the surface wave amplitude spectra is shown in Figure 2.

Assuming that we know the relevant propagation characteristics of the medium, equation (6) provides a system of equations for the five parameters defined above $(\varphi, h, \mathbf{T}, \mathbf{P})$. We define a grid in the space of these five parameters and use equation (6) to calculate the amplitude spectra of the surface waves at the points of observation for every possible combination of values of the variable parameters. Comparison of the calculated and observed amplitude spectra gives us a surface wave residual $\varepsilon_{s w}^{(i)}$ for both Rayleigh and Love waves at each frequency $\omega$. The index $i$ runs through the entire set of observations. Specifically, it corresponds to a certain station, wave type, and frequency. Let $u^{(i)}(\mathbf{x}, \omega)$ be any 
Table 1

Observed seismograms and models used for surface wave calculations for each station

\begin{tabular}{|c|c|c|c|}
\hline Station & Azimuth (o) & Distance (o) & Model \\
\hline ABKT & 271 & 24 & Tectonic \\
\hline ANTO & 287 & 42 & Tectonic \\
\hline ARU & 317 & 25 & Stable \\
\hline $\mathrm{BNG}$ & 260 & 73 & Stable \\
\hline BRVK & 319 & 17 & Stable \\
\hline CHTO & 156 & 24 & Stable \\
\hline DPC & 306 & 49 & Stable \\
\hline ENH & 118 & 20 & Stable \\
\hline ERM & 71 & 40 & Stable \\
\hline HIA & 60 & 23 & Stable \\
\hline HYB & 204 & 26 & Stable \\
\hline GNI & 282 & 33 & Tectonic \\
\hline INU & 83 & 38 & Stable \\
\hline KEV & 333 & 42 & Stable \\
\hline KIV & 290 & 34 & Tectonic \\
\hline KMI & 140 & 20 & Tectonic \\
\hline KONO & 320 & 50 & Stable \\
\hline $\mathrm{LZH}$ & 111 & 13 & Tectonic \\
\hline MA2 & 43 & 42 & Stable \\
\hline MAJO & 81 & 38 & Stable \\
\hline MDJ & 70 & 29 & Stable \\
\hline NIL & 242 & 15 & Tectonic \\
\hline NRIL & 0 & 28 & Stable \\
\hline $\mathrm{OBN}$ & 310 & 37 & Stable \\
\hline SEY & 38 & 42 & Stable \\
\hline TATO & 111 & 31 & Stable \\
\hline TLY & 40 & 14 & Tectonic \\
\hline YAK & 36 & 32 & Stable \\
\hline
\end{tabular}

observed value of the spectrum, $i=1, \ldots, N$, and $\varepsilon_{s w}^{(i)}$ be the corresponding residual of $\left|u^{(i)}(\mathbf{x}, \omega)\right|$. We define the normalized amplitude residual by the formula

$$
\varepsilon_{s w}(h, \varphi, \mathbf{T}, \mathbf{P})=\left[\left(\sum_{i=1}^{N} \varepsilon^{(i)_{s w}^{2}}\right) /\left(\sum_{i=1}^{N}\left|u^{(i)}(\mathbf{x}, \omega)\right|^{2}\right)\right]^{1 / 2} .
$$

$M_{0}$ is found by the least-squares minimization of $\varepsilon_{s w}$ for each combination of values of the five other variable parameters.

A well-known problem in estimating moment tensors from surface waves is that the radiation pattern of surface wave amplitude spectra is symmetric with respect to the epicenter. Another uncertainty in moment tensor estimation appears when source depth is much less than the wavelength of the observed surface waves, in which case the surface waves provide only three linear 
Table 2

Crustal and upper mantle models used for surface wave calculations

\begin{tabular}{|c|c|c|c|}
\hline$h(\mathrm{Km})$ & $V_{p}(\mathrm{~km} / \mathrm{s})$ & $V_{s}(\mathrm{~km} / \mathrm{s})$ & $\rho\left(\mathrm{g} / \mathrm{cm}^{3}\right)$ \\
\hline \multicolumn{4}{|l|}{ Lop Nor } \\
\hline 1.1 & 5.08 & 2.9 & 2.26 \\
\hline 5.8 & 5.90 & 3.5 & 2.59 \\
\hline 14.1 & 6.06 & 3.57 & 2.65 \\
\hline 30.9 & 6.51 & 3.77 & 2.83 \\
\hline 8.1 & 8.09 & 4.47 & 3.38 \\
\hline 20.0 & 8.07 & 4.46 & 3.38 \\
\hline 35.0 & 7.99 & 4.37 & 3.37 \\
\hline 35.0 & 7.97 & 4.36 & 3.37 \\
\hline 35.0 & 7.95 & 4.34 & 3.36 \\
\hline 35.0 & 7.93 & 4.33 & 3.36 \\
\hline 45.0 & 8.56 & 4.60 & 3.45 \\
\hline 45.0 & 8.65 & 4.64 & 3.48 \\
\hline 45.0 & 8.74 & 4.67 & 3.50 \\
\hline 45.0 & 8.82 & 4.70 & 3.53 \\
\hline 50.0 & 9.22 & 4.95 & 3.76 \\
\hline 50.0 & 9.47 & 5.09 & 3.82 \\
\hline 50.0 & 9.73 & 5.23 & 3.88 \\
\hline 50.0 & 9.98 & 5.38 & 3.94 \\
\hline 50.0 & 10.14 & 5.46 & 3.98 \\
\hline \multicolumn{4}{|l|}{ Stable } \\
\hline 19.0 & 6.14 & 3.55 & 2.74 \\
\hline 19.0 & 6.58 & 3.80 & 3.00 \\
\hline 12.0 & 8.20 & 4.65 & 3.32 \\
\hline 10.0 & 8.17 & 4.62 & 3.34 \\
\hline 10.0 & 8.14 & 4.57 & 3.35 \\
\hline 10.0 & 8.10 & 4.51 & 3.36 \\
\hline 10.0 & 8.07 & 4.46 & 3.37 \\
\hline 10.0 & 8.02 & 4.41 & 3.38 \\
\hline 25.0 & 7.93 & 4.37 & 3.39 \\
\hline 25.0 & 7.85 & 4.35 & 3.41 \\
\hline 25.0 & 7.89 & 4.36 & 3.43 \\
\hline 25.0 & 7.98 & 4.38 & 3.46 \\
\hline 25.0 & 8.10 & 4.42 & 3.48 \\
\hline 25.0 & 8.21 & 4.46 & 3.50 \\
\hline 50.0 & 8.38 & 4.54 & 3.53 \\
\hline 50.0 & 8.62 & 4.68 & 3.58 \\
\hline 50.0 & 8.87 & 4.85 & 3.62 \\
\hline 50.0 & 9.15 & 5.04 & 3.69 \\
\hline 50.0 & 9.45 & 5.21 & 3.82 \\
\hline \multicolumn{4}{|l|}{ Tectonic } \\
\hline 20.0 & 6.14 & 3.55 & 2.74 \\
\hline 20.0 & 6.80 & 3.93 & 3.00 \\
\hline 10.0 & 8.10 & 4.51 & 3.32 \\
\hline 10.0 & 8.10 & 4.51 & 3.34 \\
\hline 10.0 & 8.10 & 4.51 & 3.35 \\
\hline 10.0 & 8.10 & 4.51 & 3.36 \\
\hline
\end{tabular}

$h$ is the layer thickness 
Table 3

Information on the 10 events used in the present study

\begin{tabular}{ccccccccc}
\hline Date & Event type & $m_{b}$ & $m_{s}$ & $\begin{array}{c}\text { Number of } \\
\text { records } \\
\text { used }\end{array}$ & $\begin{array}{c}\text { Double- } \\
\text { couple } \\
\text { depth }(\mathrm{km})\end{array}$ & $M_{0}(\mathrm{~N} \cdot m)$ & $\psi(\mathrm{deg})$. & $\varphi(\mathrm{deg})$. \\
\hline 21.01 .90 & earthquake & 4.6 & - & 10 & 31 & $0.15 \cdot 10^{17}$ & 72 & 0 \\
26.05 .90 & earthquake & 5.4 & - & 5 & 3 & $0.42 \cdot 10^{16}$ & 52 & 0 \\
26.12 .94 & earthquake & 4.6 & - & 10 & 17 & $0.15 \cdot 10^{16}$ & 56 & 0 \\
16.08 .90 & explosion & 6.2 & - & 7 & 1 & $0.19 \cdot 10^{17}$ & 62 & $10-30$ \\
21.05 .92 & explosion & 6.5 & 5.0 & 16 & 3 & $0.62 \cdot 10^{17}$ & 64 & $10-15$ \\
05.10 .93 & explosion & 5.9 & 4.7 & 10 & 0 & $0.75 \cdot 10^{16}$ & 56 & $0-15$ \\
10.06 .94 & explosion & 5.8 & - & 13 & 1 & $0.52 \cdot 10^{16}$ & 64 & $20-40$ \\
07.10 .94 & explosion & 6.0 & - & 11 & 1 & $0.18 \cdot 10^{17}$ & 60 & $25-35$ \\
15.05 .95 & explosion & 6.1 & 5.0 & 9 & 3 & $0.15 \cdot 10^{17}$ & 62 & $40-60$ \\
08.06 .96 & explosion & 5.9 & 4.3 & 12 & 3 & $0.59 \cdot 10^{16}$ & 58 & $30-60$ \\
\hline
\end{tabular}

$\psi$ is the azimuth of the horizontal compression axis, $\varphi$ is the isotropic angle which determines the ratio of the isotropic and nonisotropic (double-couple) moments.

constraints on the source parameters. To improve the resolution of the source parameters, we also use the polarities of $P$-wave first arrivals. In calculating the radiation pattern of $P$ waves for a set of source parameters, we assume that the waves radiated by the isotropic (i.e., explosion) and nonisotropic (i.e., tectonic release or earthquake) source arrive simultaneously. This assumption can be abolished if the observed signs of the $P$-first arrivals would be substituted by polarities measured from long-period $P$-wave spectra (BuKCHIN et al., 1997). Before inversion we apply a smoothing procedure to the observed polarities as follows. A set of observed polarities ( +1 for compression and -1 for dilatation) radiated in nearly the same direction is combined to form a single cluster and is assigned the mean of the cluster direction. If the number of one polarity in the cluster is significantly larger than the other, we prescribe this polarity to the mean direction. If neither of the two polarities dominate, then the entire cluster is discarded.

Using the misfit between the calculated and observed polarities, we calculate a joint residual of surface wave amplitude spectra and polarities of $P$-wave first arrivals. Let $\varepsilon_{p}$ be the residual of $P$-wave first arrival polarities (the number of wrong polarities divided by the full number of observed polarities), we then define the joint residual $\varepsilon$ as the sum of the individuals

$$
\varepsilon=1-\left(1-\varepsilon_{p}\right)\left(1-\varepsilon_{s w}\right) .
$$

If both residuals $\varepsilon_{p}$ and $\varepsilon_{s w}$ are small, then $\varepsilon$ is equal approximately to their sum. We consider the values of parameters that minimize $\varepsilon$ as estimates of these parameters. 


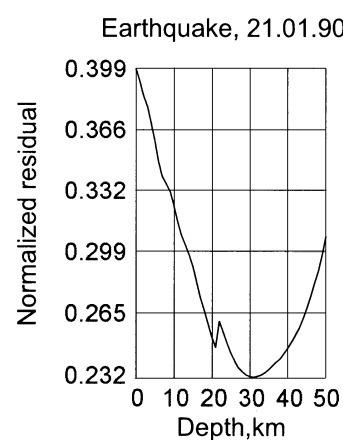

Explosion, 05.10.93

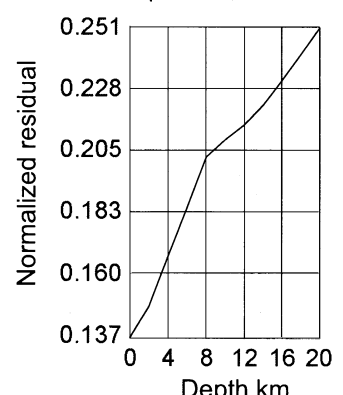

Earthquake, 26.05.90

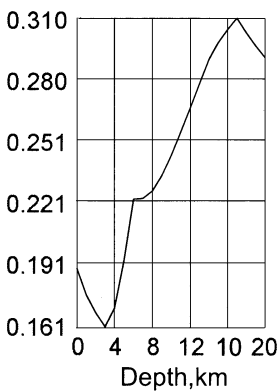

Explosion, 10.06.94

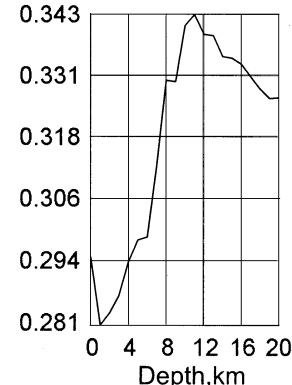

Earthquake, 26.12.94

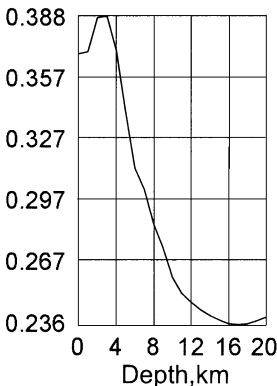

Explosion, 07.10.94

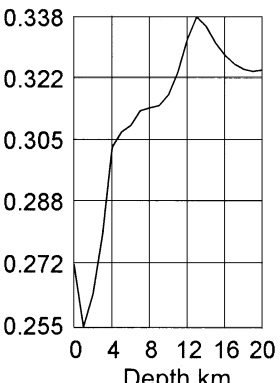

Explosion, 16.08.90

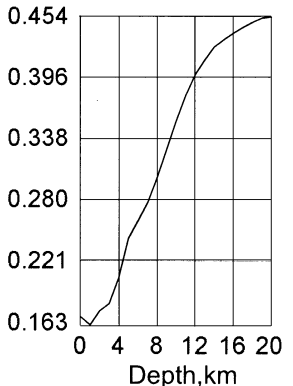

Explosion, 15.05.95

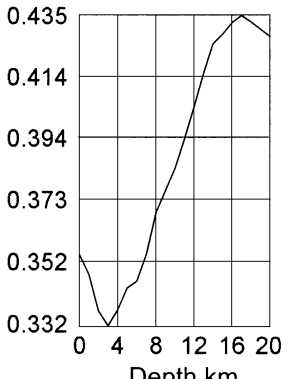

Explosion, 21.05.92

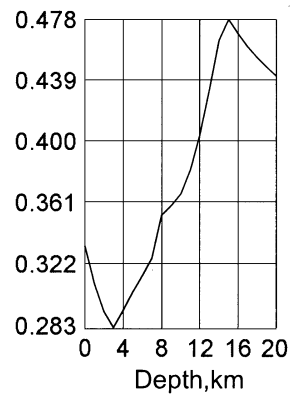

Explosion, 08.06.96

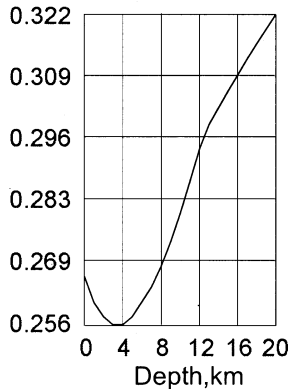

Figure 3 
Main tension axis

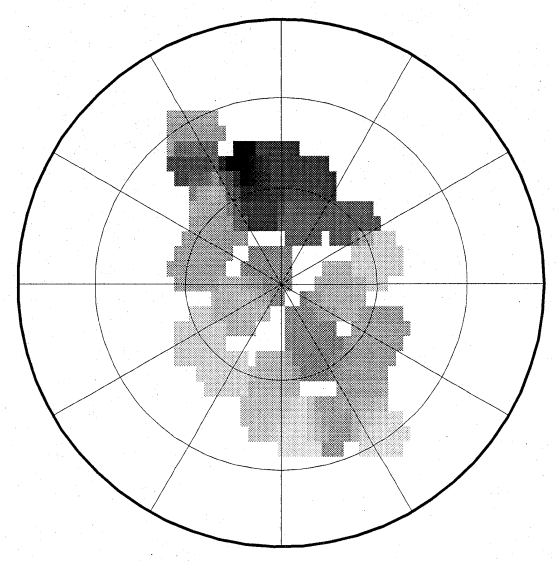

Main compression axis
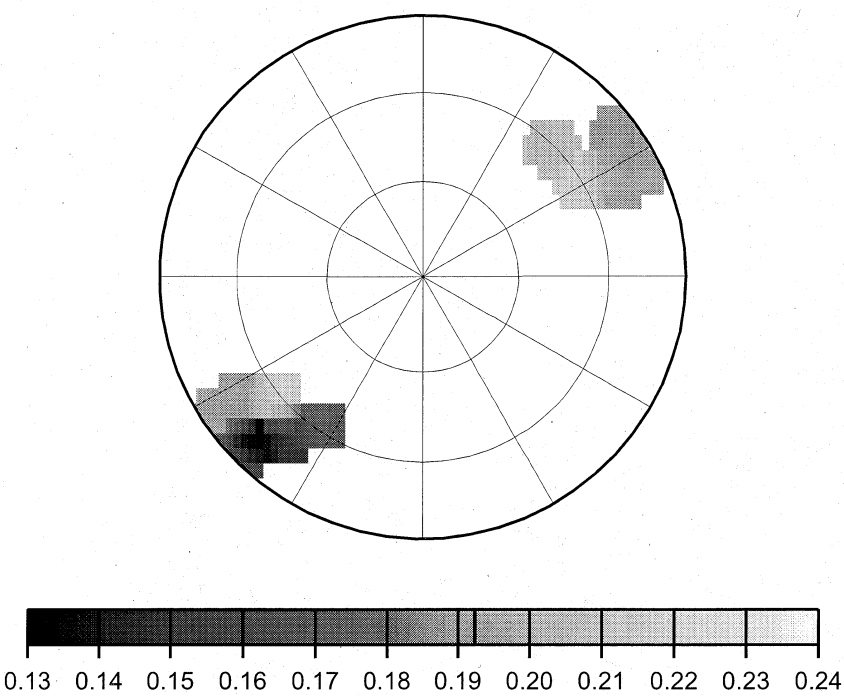

Figure 4

Partial residual functions of the orientation of the principal tension and compression axes for the doublecouple focal mechanism of the explosion on 5 October, 1993. The orientation of every axis is shown using a stereographic projection on the lower hemisphere.

To evaluate the uncertainty of each of these source characteristics we calculate partial residual functions $\varepsilon_{h}(h), \varepsilon_{\varphi}(\varphi), \varepsilon_{T}(\mathbf{T}), \varepsilon_{P}(\mathbf{P})$ in the following way. For each value of a given parameter we find among all possible combinations of the other parameters such a value which provides the minimum of this function. Repeating the 

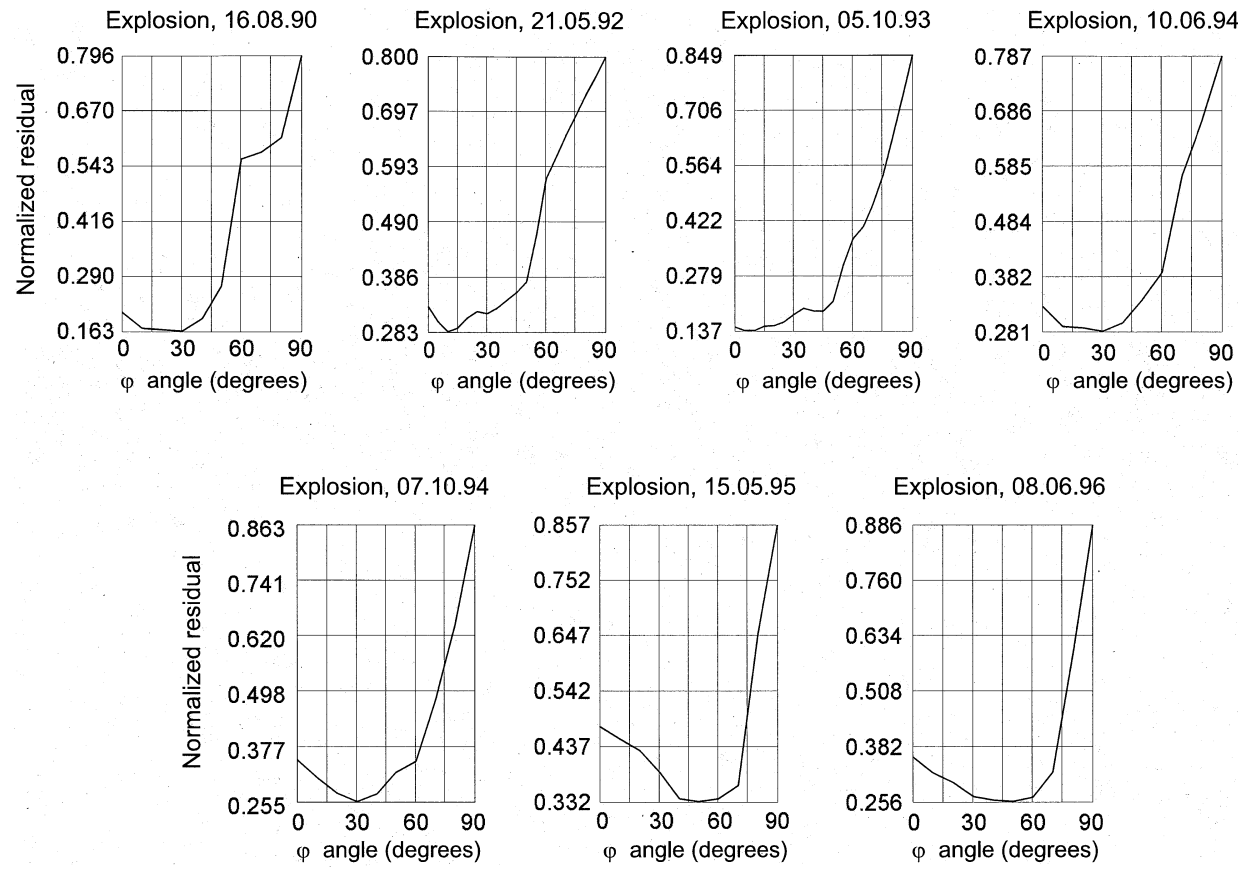

Figure 5

Partial residual functions of isotropic angle $\varphi$ for the explosions. This angle determines the ratio of seismic moments of isotropic and double-couple components.

search for all possible values of the evaluated parameter we find one at which the corresponding residual function attains its minimum and take this value as an estimate of this parameter. The behavior of the residual function characterizes the uncertainty in determining the corresponding parameter.

\section{Data and Data Processing}

We utilized IRIS and GEOSCOPE broadband digital seismograms and the ISC bulletins for 14 events that occurred on the Lop Nor test site in China from 1990 through 1996. Eight of these events are nuclear explosions, the other six are earthquakes. However, only 10 events with high signal-to-noise surface waves recorded at several stations were selected for study. The location of the selected events (7 explosions and 3 earthquakes) is given in Figure 1. The list of stations, with their distances and azimuths from Lop Nor test site, are given in Table 1.

In our calculations of the theoretical surface wave spectra we used three different models for (1) the structure for the source region, (2) the stations deployed in the 

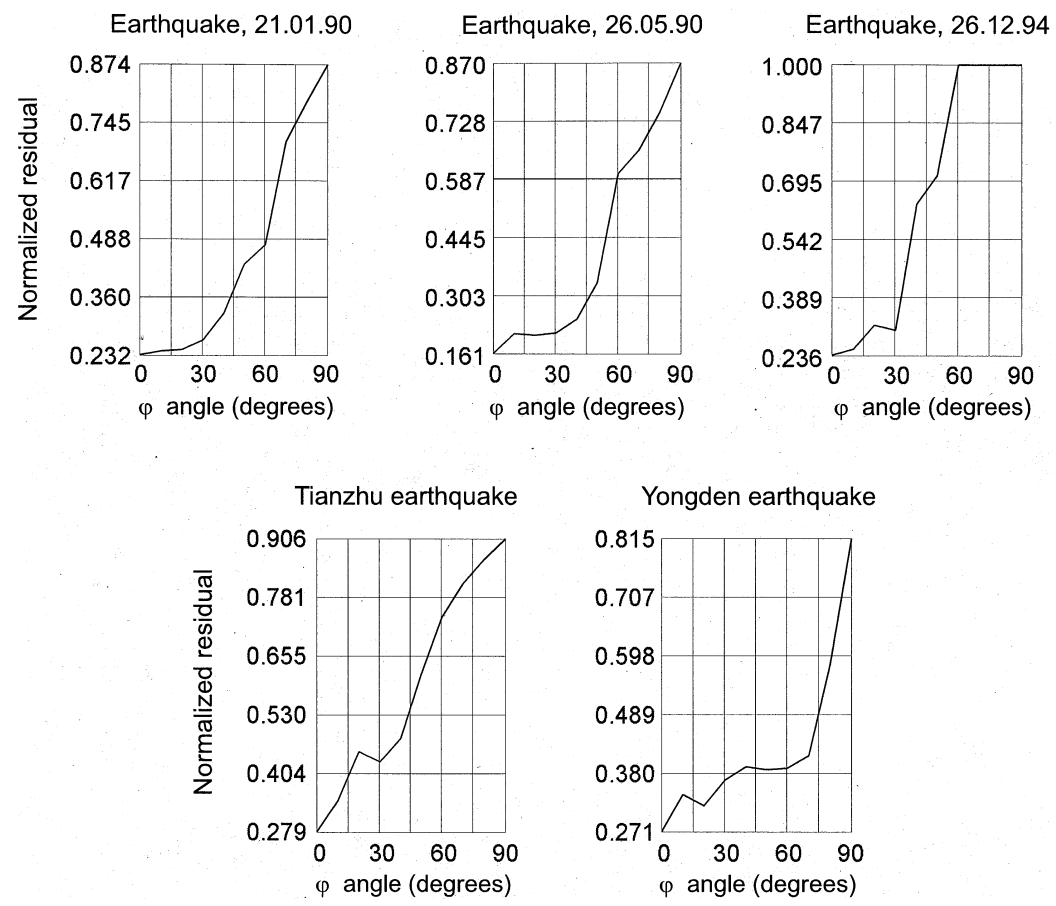

Figure 6

Same as Figure 5, but for the earthquakes.

stable continental regimes, and (3) the stations in the tectonic regions. For brevity we will call the corresponding models "Lop Nor," "Stable," and "Tectonic." The parameters for each model are presented in Table 2. For the Lop Nor model we used a regional velocity model for the four upper layers and the PREM model for deeper structure (see LASSERRE et al., 2000). The Tectonic and Stable models are identical below the first five layers. Column 4 in Table 1 indicates the model used for each station.

We estimated the source parameters using the spectra of Love and Rayleigh fundamental waves for periods ranging from $20 \mathrm{~s}$ to $70 \mathrm{~s}$. Love and Rayleigh fundamental modes were extracted by using frequency-time analysis (FTAN) and floating filtering (e.g., LANDER, 1989, and LEVSHIN et al., 1994). We also analyzed the polarization of filtered surface waves using the technique described in LANDER (1989), PAulssen et al., (1990), Levshin et al. (1992, 1994), and Levshin and RitzWOLLER (1995). Only records in which the surface wave polarization pattern did not exhibit significant azimuthal anomalies $\left(\leq 15^{\circ}\right)$ were used for further analysis. Examples of normalized amplitude spectra are shown in Figure 2. Note that the amplitude of the Love waves, which cannot be excited by a pure isotropic source, is comparable with the amplitude of the Rayleigh waves. 
(a)

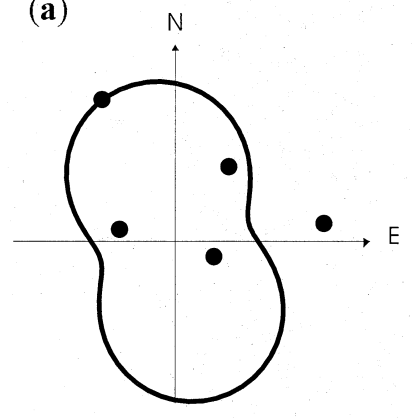

(c)

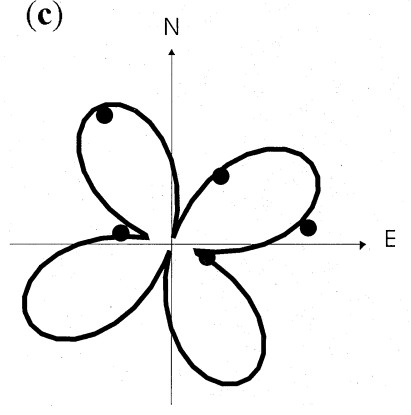

(b)

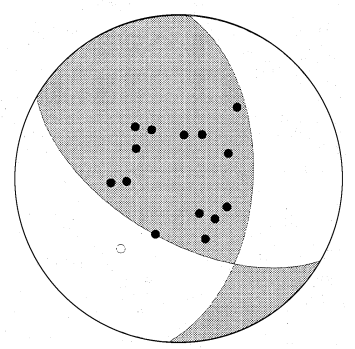

(d)

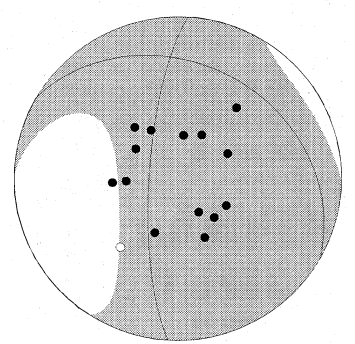

Figure 7

Comparison of observed Rayleigh wave spectral amplitudes at $26 \mathrm{~s}$ period and polarities of first arrivals for the explosion on June 10, 1994 with those predicted for two different source models: (a, b) - pure doublecouple; (c, d) - non-zero isotropic component is added. (a, c) - observed (filled circles) and predicted (solid line) surface wave spectral amplitudes; (b, d) - observed positive (dark dots) and negative (white dots)

$P$-wave polarities; predicted positive (shaded areas) and negative (white areas) $P$-wave polarities.

\section{Results of Inversions}

A summary of the inversion results is given in Table 3.

\subsection{Double-couple Depths and Focal Mechanisms}

The focal mechanisms for the earthquakes and for the double-couple components of the explosions are shown in Figure 1.

The double-couple depth is well resolved for all events studied and varies from 0 to $3 \mathrm{~km}$ for the explosions and for the earthquakes we obtained depths of $3 \mathrm{~km}$, $31 \mathrm{~km}$, and $17 \mathrm{~km}$. The partial residual function of double-couple depth is presented in Figure 3 for all events.

Figure 4 shows an example of the resolution of the double-couple focal mechanism for the large explosion that occurred on October 5, 1993 with $m_{b}=5.9$ and $m_{s}=4.7$. This figure displays the partial residual function of principal axes 

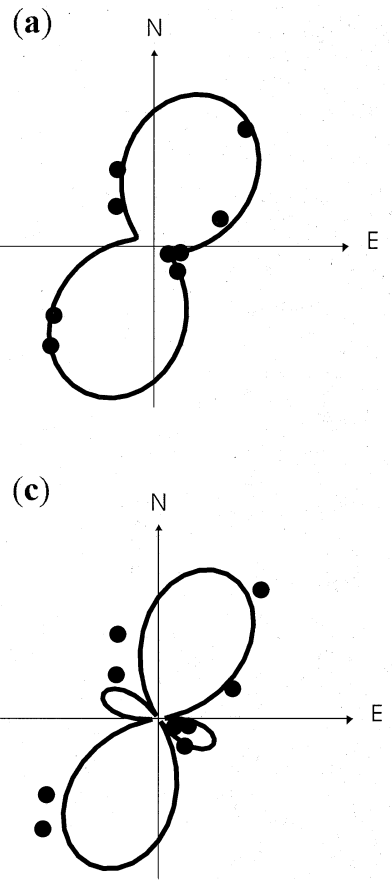

(b)

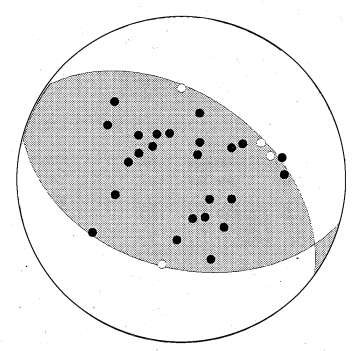

(d)

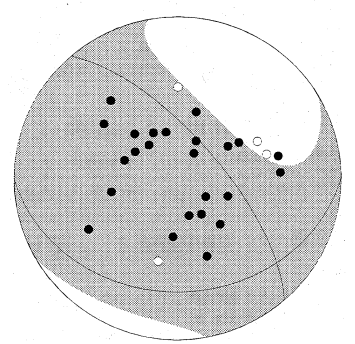

Figure 8

Same as Figure 7, but for the Yongden earthquake in Gansu province, China, on July 21, 1995 and $50 \mathrm{~s}$ period.

orientation. The principal compression axis is resolved better than the principal tension axis.

The number of surface wave records that we selected for the studied events was admittedly very small (see column 5 in Table 3), particularly for the earthquakes on the test site. For some events, only a few polarities of $P$-wave first arrivals were available. As a result, the double-couple focal mechanism was very well resolved not only for the earthquake on 21.01.90, but also for half of the events the principal compression axis, which trends in the SE-NW direction, was resolved well. Fortunately, GAO and RICHARDS (1994) estimated focal mechanisms of several earthquakes in the vicinity of Lop Nor. Considering the focal mechanisms of the studied events, and the solutions obtained by Gao and Richards, we found that while focal mechanisms vary widely, there is a moment tensor characteristic which is quite stable for all events. This characteristic is the orientation of the principal axes of the $2 \times 2$ minor of the moment tensor corresponding to the horizontal coordinates $\left\{M_{x x}, M_{x y}, M_{y x}, M_{y y}\right\}$. This minor describes the horizontal deformations on the horizontal plane. We found that for all events, horizontal compression is dominant, and the principal horizontal compression axis deviates from the average direction 
(with an azimuth of $60^{\circ}$ ) by no more than $12^{\circ}$. The direction of this axis for every event is given in the eighth column in Table 3.

\subsection{Isotropic Component}

These observations allow us to improve the estimates of the isotropic angle $\varphi$ by assuming that the orientation of the horizontal deformations is a stable characteristic for the region under study. The average direction of horizontal compression is deviated from the fault visible in Figure 1 by about $45^{\circ}$. With this assumption, we applied an a priori constraint on the possible double-couple focal mechanisms. This constraint was formulated as a condition that the azimuth of the principal compression axis of the horizontal minor of the moment tensor cannot differ from $60^{\circ}$ by more than $\pm 45^{\circ}$. This constraint is weak, but reduces the number of possible focal mechanisms in half. Although the constraint is heuristic, we can present a special case when it is exact. This is when the deviatoric tectonic stresses in the region are horizontal and the direction mentioned above is the direction of principal compression. Then the constraint follows from the principle of positive deformation energy: $\sum_{i, j=1}^{3} S_{i j} M_{i j} \geq 0$, where $\mathbf{S}$ is the regional stress tensor, and $\mathbf{M}$ is moment tensor of event in the region.

When we applied this constraint, the resolution of the isotropic angle $\varphi$ defining the seismic moments ratio was improved for three of the seven studied explosions (16.08.90, 05.10.93, and 08.06.96) but the mean of the curves did not vary appreciably. The curves of partial residual functions of the isotropic angle $\varphi$ for all explosions are shown in Figure 5. Those for the three earthquakes are displayed in Figure 6. We also applied this method to two earthquakes that occurred in the Gansu province, China (Tianzhu, 1996, $M_{s}=4.9$, depth $12 \mathrm{~km}$, and Yongden, 1995, $M_{s}=5.4$, depth $6 \mathrm{~km}$ ) studied by LASSERRE et al. (2000) under the assumption of a pure double-couple source. The partial residual functions of the isotropic angle $\varphi$ are also shown in Figure 6. The explosions, with perhaps one exception (05.10.93), all display substantial non-zero isotropic angles $\varphi$ ranging from about $10^{\circ}$ to $50^{\circ}$, corresponding to $M_{0 e x} / M_{0 q u}$ ranging from about $20 \%$ to $120 \%$. In contrast, the isotropic angles for the earthquakes are all estimated to be approximately 0 .

Figure 7 illustrates the effect of adding an isotropic component to the doublecouple source model for an explosion (on June 10, 1994). Theoretical Rayleigh wave amplitude spectra and $P$-wave first arrival polarities are compared here with observations for two different moment tensors. One (a, b) was obtained by minimizing the joint residual for a fixed zero isotropic angle (double-couple source model). The other (c, d) is a result of a similar minimization, but for a varying isotropic angle $\left(30^{\circ}\right.$ is the estimated optimal value). The fit to $P$-wave first arrival polarities is good for both source models, however the fit to Rayleigh wave amplitude spectra is decidedly better in the case of a non-zero isotropic component. 
Figure 8 illustrates the effect of adding an isotropic component for the Youngden earthquake. Similar to Figure 7, the results for two different moment tensors are presented here. One $(a, b)$ was obtained by minimizing the joint residual for a varying isotropic angle. The other $(\mathrm{c}, \mathrm{d})$ is a result of a similar minimization but for a fixed $\left(30^{\circ}\right)$ isotropic angle. In contrast with the previous case the fit is better for a pure double-couple source.

\section{Conclusions}

The results above are consistent with the hypothesis that motivates this study. Namely, that for the events we analyzed on the Lop Nor test site, surface wave amplitude spectra combined with polarities of $P$-wave first arrivals can be used to discriminate explosions from earthquakes, based on source characteristics alone (a combination of the double-couple depth and the ratio of the isotropic to nonisotropic moments). Considerably more work remains to be done, however, to determine if the method can be applied to events with much smaller moments and if the method is transportable to other regions.

\section{Acknowledgments}

This research was supported by a NATO Linkage Grant N 9500775 between the University of Colorado at Boulder and the International Institute of Earthquake Prediction Theory and Mathematical Geophysics, Moscow, by a subcontract with the Department of Geological Sciences at Cornell University under EAR-9804859 from the US National Science Foundation, by the Russian Foundation of Fundamental Research Project 99-05-64964, the International Science and Technology Center Project 1293-99, by DSWA contract DSWA01-97-C-0157 to the University of Colorado at Boulder, by the US Arms Control and Disarmament Agency. We thank Alexander Lander and Howard Patton for helpful discussions and Istvan Bondar and Nikolai Shapiro for valuable reviews. Thanks to the IRIS-DMC and GEOSCOPE staffs for providing digital data.

\section{REFERENCES}

AKI, K., and Tsai, Y. (1972), The Mechanism of Love Wave Excitation by Explosive Sources, J. Geophys. Res. 77, 1452-1475.

Archambeau, C. B. (1972), The Theory of Stress Wave Radiation from Explosions in Prestressed Media, Geophys. J. 29, 329-366.

Babich, V. M., Chikachev, B. A., and Yanovskaya, T. B. (1976), Surface Waves in a Vertically Inhomogeneous Elastic Half-space with Weak Horizontal Inhomogeneity, Izv. Akad. Nauk SSSR, Fizika Zemli 4, 24-31. 
Brune, J. N., and Pomeroy, P. W. (1963), Surface Wave Radiation Patterns for Underground Nuclear Explosions and Small-magnitude Earthquakes, J. Geophys. Res. 68, 5005-5028.

Bukchin, B. G. (1990), Determination of Source Parameters from Surface Waves Recordings Allowing for Uncertainties in the Properties of the Medium, Izv. Akad. Nauk SSSR, Fizika Zemli 25, 723-728.

Bukchin, B. G., Lander, A. V., Mostinsky, A. Z., and Maksimov, V. I. (1997), Determination of Seismic Source Parameters by Analysis of Coherence of Body Wave Phases. Theoretical Problems in Geophysics. 29, Moscow (Comput. Seismol.) In (eds. V. I. Keilis-Borok, and G. M. Molchan,) 3-17, 1997 (in Russian). English translation: Comput. Seismology and Geodynamics 4, AGU, 2001, in press.

Burger, R., Lay, T., Wallace, T., and Burdick, L. (1986), Evidence of Tectonic Release in Long-period S Waves from Underground Nuclear Explosions at the Novaya Zemlya Test Sites, Bull. Seismol. Soc. Am. 76, 733-755.

Day, S. M., and Stevens, J. L. (1986), An Explanation for Apparent Time Delays in Phase-reversed Rayleigh Waves from Underground Nuclear Explosions, Geophys. Res. Lett. 13, 1423-1425.

Day, S. M., Cherry, J. T., Rimer, N., and Stevens, J. L. (1987) Nonlinear Model of Tectonic Release from Underground Explosions, Bull. Seism. Soc. Am. 77, 996-1016.

Ekström, G., and Richards, P. G. (1994), Empirical Measurements of Tectonic Moment Release in Nuclear Explosions from Teleseismic Surface Waves and Body Waves, Geophys. J. Int. 117, 120-140.

GaO, L.-P., and Richards, P. G. Studies of Earthquakes on and near the Lop Nor, China, nuclear test site, In Proc. of the 16th Annual Seismic Research Symposium, 7-9 Sept. 1994, 106-112, Phillips Lab., Directorate of Geophysics, 1994.

Harkrider, D. G., Stevens, J. L., and Archambeau, C. B. (1994), Theoretical Rayleigh and Love Waves from an Explosion in Prestressed Source Regions, Bull. Seismol. Soc. Am. 84, 1410-1442.

Helle, H. B., and RygG, E. (1984), Determination of Tectonic Release from Surface Waves Generated by Nuclear Explosions in Eastern Kazakhstan, Bull. Seismol. Soc. Am. 74, 1883-1898.

LANDER, A. V., Frequency-time analysis. In Seismic Surface Waves in a Laterally Inhomogeneous Earth. (V.I. Keilis-Borok, ed.), (Kluwer Acad. Publ., Dordrecht, 1989) pp. 153-163.

Lasserre, C., Bukchin, B., Bernard, P., Tapponier, P., Gaudemer, Y., Mostinsky, A., and Rong DAILU (2001), Source Parameters and Tectonic Origin of the June 1, 1996 Tianzhu ( $M_{w}=5.2$ and July 21, 1995 Yongden $\left(M_{w}=5.6\right)$ Earthquakes, near Haiyuan Fault (Gansu, China), Geophys. J. Intl. 144, 206-220.

Levshin, A. L. (1985), Effects of Lateral Inhomogeneity on Surface Wave Amplitude Measurements, Annales Geophysicae 3(4), 511-518.

Levshin, A. L., Yanovskaya, T. B., Lander, A. V., Bukchin, B. G., Barmin, M. P., Ratnikova, L. I., and Its, E. N. Seismic Surface Waves in Laterally Inhomogeneous Earth (ed. V. I. Keilis-Borok) (Kluwer. Publ., Dordrecht, 1989)

Levshin, A. L., Ratnikova, L. I., and Berger, J. (1992), Peculiarities of Surface Wave Propagation across the Central Eurasia, Bull. Seismol. Soc. Am. 82, 2464-2493.

Levshin, A., Ritzwoller M., and Ratnikova, L. (1994), The Nature and Cause of Polarization Anomalies of Surface Waves Crossing Northern and Central Eurasia, Geophys. J. Int. 117, 577-591.

Levshin, A. L., and Ritzwoller, M. H. (1995), Charactersistics of Surface Wave Generated by Events on and near the Chinese Nuclear Test Site, Geophys. J. Intl. 123, 131-148.

Li, Y., Toksöz, M. N., and Rodi, W. (1995), Source Time Functions of Nuclear Explosions and Earthquakes in Central Asia Determined Using Empirical Green's Functions, J. Geophys. Res. 100, 659-674.

Masse, R. P. (1981), Review of Seismic Source Models for Underground Nuclear Explosions, Bull. Seismol. Soc. Am. 71, 1249-1268.

Mueller, R. A., and Murphy, J. R. (1971), Seismic Characteristics of Underground Nuclear Detonations. Part I: Seismic Spectrum Scaling, Bull. Seismol. Soc. Am. 61, 1675-1692.

Patton, H. J. Seismic moment estimation and the scaling of the long-period explosion source spectrum. In Explosion Source Phenomenology, Geophysical Monograph 65, AGU (eds. S. R. TAYLOR, H. J. PATtON, and P. G. Richards) pp. 171-184, 1991.

Paulssen, H., Levshin, A. L., Lander, A. V., and Snieder, R. (1990), Time and Frequency Dependent Polarization Analysis: Anomalous Surface Wave Observations in Iberia, Geophys. J. Int. 103, 483-496.

Press, F., and Archambeau, C. B., (1962) Release of Tectonic Strain by Underground Explosions, J. Geophys. Res. 67, 337-343. 
Stevens, J. L. (1986), Estimation of Scalar Moments from Explosion-generated Surface Waves, Bull. Seismol Soc. Am. 76, 123-151.

Stevens, J. L., Barker, T. G., Day, S. M., Mclaughlin, K. L., Rimer N., and Shkoller, B. Simulation of teleseismic body waves, regional seismograms, and Rayleigh wave phase shifts using two-dimensional nonlinear models of explosion sources. In Explosion Source Phenomenology, Geophysical Monograph 65, $A G U$ (eds. S. R. Taylor, H. J. Patton, and P. G. Richards) pp. 239-252, 1991.

Toksöz, M. N., and Kehrer, H. H. (1972), Tectonic Strain Release by Underground Nuclear Explosions and its Effect on Seismic Discrimination, Geophys. J. R. Astr. Soc. 31, 141-161.

Wallace, T. C., Helmberger, D. V., and Engen, G. R. (1985), Evidence of Tectonic Release from Underground Nuclear Explosions in Long-period S Waves, Bull. Seismol. Soc. Am. 76, 123-151.

Wallace, T. C., Body wave observations of tectonic release. In Explosion Source Phenomenology, Geophysical Monograph 65, AGU (eds. S. R. TAYlor, H. J. PAtton, and P. G. Richards) pp. 161-170, 1991.

Wallace, T. C., and Tinker, M. A. (1996), The Last Nuclear Weapons Test? A Brief Review of the Chinese Nuclear Weapons Program, IRIS Newsletter 15 (3).

Walter, W. R., and Patton, H. J., (1990) Tectonic Release from SJVE, Geophys. Res. Lett. 17, 15171520 .

Woodhouse, J. H. (1974), Surface Waves in the Laterally Varying Structure, Geophys. J. R. Astr. Soc. 90, 713-728.

ZHANG, J. Polarization characteristics of seismic waves from the May 21, 1992 Lop Nor nuclear explosion using IRIS/GSN broadband data. In Proc. of the 16th Annual Seismic Research Symposium, 7-9 Sept. 1994, 393-397, Phillips Lab., Directorate of Geophysics, 1994.

(Received June 25, 1999, revised March 20, 2000, accepted May 15, 2000)

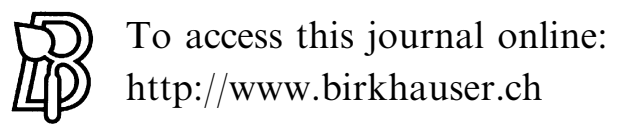

